\title{
Vibration Analysis of Composites
}

\author{
Prashant Mahale*, Shrutika Karpe, Shubhada Khedkar and Kajal Khule \\ Mechanical Engineering, Savitribai Phule Pune University, Pune
}

Accepted 02 March 2016, Available online 15 March 2016, Special Issue-4 (March 2016)

\begin{abstract}
Laminated composite plate structures find numerous applications in aerospace, military and automotive industries. The role of transverse shear is very important in composites, as the material is weak in shear due to its low shear modulus compared to extensional rigidity. Hence, an accurate understanding of their structural behaviour is required, such as deflections and stresses. In this paper, a number of finite element analyses have been carried out for various side-to-thickness ratios, aspect ratios and modulus ratios to study the effect of transverse shear deformation on deflection and stresses of laminated composite plates subjected to uniformly distributed load. The numerical results found out, on the deflections and stresses, that the effect of coupling is to decrease the deflections with the increase in the aspect ratio and modulus ratio and increase the stresses with the increase in the side-to-thickness ratio and modulus ratio.
\end{abstract}

\section{Keywords: Vibration, Composites, Analysis, Central Deflection}

\section{Introduction}

The classical plate/shell theory which is adequate only for thin shells. However, the linear layer structural shell element allows to model thin to moderately thick plate and shell structures with a side-to-thickness ratio of roughly 10 or greater. The plates studied here is 25 mm thick and especially for $\mathrm{a} / \mathrm{h}=10$ to 40 , the thin shell model as well as element should not be used. Since in thick plates, the bending and through-thickness transverse shear stresses are dominant than membrane stress, which are not captured by the thin plate model where the thickness and out of plane stresses are assumed negligible.

Of sixteen ply simply supported symmetric laminated composite plate; it was observed that, the deflections are larger for smaller modulus ratios and aspect ratios, the degree of orthotropy has less influence on the deflections for large ratios of E1/E2, the effect of shear deformation is to decrease the deflections and increase the stresses with the increase of modulus ratio sand side-to-thickness ratios.

\section{Failure Analysis of Laminated Composite Beams by Finite Element Method}

In this study, a four noded rectangular element with 5 degree of freedom (DOF) has been taken for the failure analysis of laminated composite beams. Apart from this the static and free vibration analysis of homogenous
Timoshenko beam with two noded bar element having two DOF in each node with different boundary conditions are presented in MATLAB and compared with the results available in literature, and it is observed that the present results show the errors less than $1 \%$. Natural frequency up to fifteen modes are compared for both Simply supported (SS) and ClamedClamed (CC) boundary conditions and also it is compared with different elements for two nodes, it has been found that with the increase of elements.

The present results are coming more closure to exact.

Examples have been worked out to validate the proposed approach. A number of examples have been presented and comparisons have been made with the results of earlier investigators wherever possible. The examples include isotropic and laminated composite beams with various boundary conditions. Two noded bar element with two degrees of freedom and eightnoded beam element is considered with five degrees of freedom per node.

Example 1 Results for Central deflection and Nondimensional Natural frequencies for Timoshenko beam clamped at both ends for different meshing (h/l=0.01) are shown in table.

Table 1 (a) Central deflection and (b) Nondimensional Natural frequencies for a Timoshenko Beam clamped at both ends for different meshing $(\mathrm{h} / \mathrm{l}=0.01)$ 
Table 1 (a)

\begin{tabular}{|c|c|c|}
\hline Mesh size & Present Result & Exact Result \\
\hline $10 \times 1$ & 0.0149578 & \\
\hline $20 \times 2$ & 0.014987 & \multirow{2}{*}{0.015} \\
\hline $40 \times 4$ & 0.0149919 & \\
\hline $60 \times 6$ & 0.0149925 & \\
\hline
\end{tabular}

Table 1 (b)

\begin{tabular}{|c|c|c|c|}
\hline \multicolumn{4}{|c|}{ Mesh Size v/s Non Dimensional Natural Frequencies } \\
\hline Mesh Size & Mode 1 & Mode 2 & Mode 3 \\
\hline 10x1 & 2.9974 & 9.0123 & 9.2333 \\
\hline $20 \times 2$ & 2.9702 & 8.718 & 9.4322 \\
\hline $40 \times 4$ & 2.96666 & 8.5720292 & 9.607289 \\
\hline $60 \times 6$ & 2.96623 & 8.81063 & 9.29782 \\
\hline
\end{tabular}

Based on the study following conclusions has been drawn:

1. Timoshenko beam (C.C. and S.S.) gives better results for non-dimensional natural frequencies when aspect ratios decrease.

2. Central deflection of clamped beam $(\mathrm{h} / \mathrm{l}=0.01)$ is minimum for the lamination scheme of $0 / 30$.

3. Ultimate strength of clamped beam $(\mathrm{h} / \mathrm{l}=0.01)$ is maximum for the lamination scheme of $0 / 30$.

\section{Theoretical and Experimental Modal Analysis of Laminated Composite Beams}

In this paper modal analysis of laminated composite beams is carried out using both theory and experiment. In the theoretical part of the investigation, the dynamic stiffness method is utilized when predicting the natural frequencies and mode shapes of a range of laminated composite beams. These results are validated by modal identification techniques that are well established using impulse hammer kit.

The free vibrational characteristics of structures are of considerable importance in engineering design. As a consequence a huge amount of research work has been expended to facilitate both the theoretical as well as experimental determination of-free vibrational behaviour of structures commonly known as modal analysis. In particular, the problem becomes much more difficult when dealing with fiber reinforced laminated composite structures. The difficulty arises due to the anisotropic nature of fibrous composites. The current study is focused on this specific topic wherein the modal analysis of laminated composite beams is carried out using both theoretical and experimental means. The theory used is that of the dynamic stiffness method which is well known for its model accuracy in free vibration analysis. Theoretical results are validated by experimental modal identification using the impulse hammer techniques and associated software.
Modal analysis characterizes the deformation of a structure due to external vibration input. When the structure is excited by external vibration energy, it is deformed in a number of well-defined wave-like patterns or modes. Each mode has its own specific natural frequency and mode shape and an inherent amount of damping.

Table 2 Theoretical, experimental and FEM results $\mathrm{Hz})$ for cantilever composite beams with ply orientations of 00 , and 100

\begin{tabular}{|c|c|c|c|}
\hline $\begin{array}{c}\text { Ply } \\
\text { Angle(deg) }\end{array}$ & $\begin{array}{c}\text { Theoretical } \\
\text { results } \\
\text { (Hz) }\end{array}$ & $\begin{array}{c}\text { Experimental } \\
\text { results (Hz) }\end{array}$ & $\begin{array}{c}\text { FEM } \\
\text { Analysis } \\
\text { results }\end{array}$ \\
\hline & 41.966 & 68.75 & 40.44 \\
\hline 0 & 263 & 275.62 & 262.22 \\
\hline & 308.96 & 375 & 368.21 \\
\hline & 34.832 & 34.38 & 36.28 \\
\hline 10 & 215.8 & 222.5 & 230 \\
\hline & 365.25 & 347.5 & 327.72 \\
\hline
\end{tabular}

An investigation into the modal behaviour of laminated composite beams has been carried out using both theory and experiments. The theoretical study is based on the dynamic stiffness and finite element methods whereas the experimental has been carried out using impulse hammer techniques. The theoretical and experimental results for cantilever composite beams agreed very well. However, it should be noted that the effect of damping has not been discussed in details since the damping data for the numerical model were not available. The investigation paves way for further studies on the aero elasticity of composite wings. This will be a useful extension of the present study and it is in this context the method presented in this paper is expected to be most useful.

\section{Free vibration analysis of beams by using a third- order shear deformation theory}

In this study, free vibration of beams with different boundary conditions is analyzed by using the thirdorder shear deformation theory. The boundary conditions of beams are satisfied using Lagrange multipliers. Using Lagrange's equations, the problem is reduced to the solution of a system of algebraic equations. The first six eigenvalues of the considered beams are calculated for different thickness-to-length ratios. The results are compared with the results based on Timoshenko and Euler-Bernoulli beam theories.

In the present study, free vibration of beams with different boundary conditions is analyzed based on the third-order shear deformation theory (TSDT). Frequency equations of the beams are derived using Lagrange's equations. The first six eigenvalues of the considered beams are calculated for different thickness-to-length ratios. The obtained results are compared with earlier results based on CBT and FSDT. 
The free vibrations of the beams have been investigated for different thickness-to-length ratios according to TSDT. The eigenvalues of the beams obtained with various boundary conditions are compared with the results of CBT and FSDT. Using Lagrange's equations with the trial functions in the polynomial form and satisfying the constraint conditions by the use of Lagrange multipliers is a nice way for studying the free vibration characteristics of the beams. It is observed from the investigations that the CBT, FSDT and TSDT results are close to each other for small values of $\mathrm{h} / \mathrm{L}$. However, as the thickness-tolength ratio becomes greater, the results of the classical beam theory significantly differ from others. This situation is also observed as the mode numbers increase. The frequencies of TSDT are slightly greater than that of FSDT. It is expected that the results of the third-order shear deformation theory give the closest frequency values to the exact frequency values in the considered three beam theories as it is proved for plates. The results obtained are accurate and are expected to be useful to other researchers for comparison.

\section{Modal Analysis of Fibre Reinforced Composite Beams with a Transverse Crack Using ANSYS}

In many structures like high speed machineries, aircrafts and light weight structures composite beams and beam like structures are main constituent elements.

In the present work two Fiber- Reinforced Plastic (FRP) materials, Graphite Fiber Reinforced Polyamide and E-Glass Fiber Reinforced Polymer have been selected as beam materials for modal analysis using ANSYS 13.0. The analysis is carried out for these two beams in different ways. Initially the analysis is carried out for different orientation of fibers for two beams. Later the effect of dimensions is analyzed by varying one dimension of the beam at a time by keeping the other two constant. In the next step the analysis is performed for constant dimensions of each beam for same layer orientation and constant volume fraction of fiber by introducing transverse cracks of different depths at various positions along the length of the beam. The results obtained are analyzed.

The following conclusions can be drawn from the present investigations of the composite beam having transverse open crack. The in-plane bending frequencies decrease, in general, as the fiber angle increases; the maximum frequency occurs at $\alpha=0^{\circ}$ and decrease gradually with increasing the fiber angle up to a minimum value obtained for $\alpha=90^{\circ}$. It is found that all the first three natural frequencies of composite beams of the two materials decrease as the length of the beam increases. It is observed from the first three natural frequencies obtained, the variation in natural frequency with width of the composite beams shows similar pattern for 1st and 3rd and another same pattern for 2 nd natural frequency and the variation is linear. It is also found that the height of the composite beam increases the first three natural frequencies increases. The effect of cracks is more pronounced near the fixed end than at far free end. It is concluded that the first three natural frequencies are most affected when the crack is located nearer to the fixed end, the middle of the beam and the free end, respectively. The analysis can be used to identify the presence of cracks in various composite beams by analyzing the first three frequency patterns of intact and cracked composite beams. The analysis can be carried out for different volume fractions of fibres and different boundary conditions of composite beams. The mode shapes can be extracted and the analysis of the mode shapes can be used to locate the intensity of depth and location of the crack. Various other types of analysis can be carried out using MATLAB. The vibration results obtained using ANSYS 13 can be verified by conducting experiments on composite beams using FFT analyzer for various boundary conditions.

\section{Modal Analysis of Composite Beam Reinforced by Aluminium-Synthetic Fibers with and without Multiple Cracks Using ANSYS}

Classical laminate plate theory and first order shear deformation theory are used for analysis. The assumption of displacements as linear functions of the coordinate in the thickness direction has proved to be inadequate for predicting the response of thick laminates. The results of the study leaded to conclusions that, presented method was adequate for the vibration analysis of cracked cantilever composite beams, and by using the drop in the natural frequencies and the change in the mode shapes, the presence and nature of cracks in a structure can be detected.

The model chosen is a cantilever composite beam of uniform cross-section having dimension 500x30x6 $\mathrm{mm}$. This is a three layer sandwich composite beam. The middle layer is of Synthetic fiber (Nylon or GFRP) and the upper and lower layer is of Aluminium. In this model the location of cracks will vary from $\mathrm{L} 1 / \mathrm{L}=0.1$ to $\mathrm{L} 9 / \mathrm{L}=0.9$. In order to check the natural frequency of cantilever composite beam, first we calculate the mechanical properties of synthetic fiber (Nylon and GFRP) and Aluminium, like ultimate tensile strength, yielding strength and Poisson ratio.These mechanical properties are fed into ANSYS-14 to calculate the deflection and natural frequency for composite cantilever beam.

\section{Conclusion}

1. The following conclusions can be made from this research paper:

2. The deflection of composite beams is less than that of pure material beams if nylon is taken as synthetic fiber with $\mathrm{Al}$, but if GFRP is taken then its deflection is 
found to be increased when compared to pure GFRP. So, nylon suits good to make composite beam with $\mathrm{Al}$ as compared to other synthetic fibers like GFRP.

3 . As the number of cracks increases the deflection in beam increases.

4. The natural frequencies of pure materials (GFRP \& Nylon) are larger than those of composite beams made by them

5. As the number of cracks on beams increases, the natural frequencies decrease.

6 . The natural frequency found higher in the fifth mode shape for all composite and pure materials.

\section{References}

Chandramani Mishra1, Nisheet Tiwari2 \& S.P.S. Rajput 3International Journal of Application or Innovation in Engineering \& Management (IJAIEM)

J. Njuguna, J. R. Banerjee and C.W. Cheung March 23-25, (2006)

Mesut S, I'MS,EK and Turgut Kocatu“ RK S־adhan`a Vol. 32, Part 3, June 2007. MS received 31 March 2006; revised 29 September 2006

Syed Ayesha Yasmeen, Anantha Abhijit P , Dr. D. Srinivasa Rao3 International Journal of Modern Engineering Research (IJMER)

Husain Mehdi, Rajan Upadhyay, Rohan Mehra, Adit Singhal2, (Year 2014), International Journal of Mechanical Engineering ( IJME ) Volume 4 Issue 2, ISSN : 2277-7059 